\title{
A Deep Learning Framework Based on Multisensor Fusion Information to Identify the Airplane Wake Vortex
}

\author{
Yi Ai $\mathbb{D}$, Yuanji Wang, Weijun Pan, and Dingjie Wu
}

Civil Aviation Flight University of China, China

Correspondence should be addressed to Yi Ai; aiyi@cafuc.edu.cn

Received 8 October 2021; Accepted 5 November 2021; Published 28 November 2021

Academic Editor: Gengxin Sun

Copyright (C) $2021 \mathrm{Yi}$ Ai et al. This is an open access article distributed under the Creative Commons Attribution License, which permits unrestricted use, distribution, and reproduction in any medium, provided the original work is properly cited.

\begin{abstract}
Along with the rapid improvement of the aviation industry, flight density also increases with the increase of flight demand, which directly leads to the increasingly prominent influence of wake vortex on flight safety and aviation control. In this paper, we propose a new joint framework-a deep learning framework-based on multisensor fusion information to address the detection and identification of wake vortices in the near-Earth phase. By setting multiple Doppler lidar in near-Earth flight areas at different airports, a large number of accurate wind field data are captured for wake vortex detection. Meanwhile, the airport surveillance radar is used to locate the wake vortex. In the deep learning framework, an end-to-end CNN-LSTM model has been employed to identify the airplane wake vortex from the data detected by Doppler lidar and the airport surveillance radar. The variables including the wind field matrix, positioning matrix, and the variance sequence are used as inputs to the CNN channel and LSTM channel. The identification and location information of the wake vortex in the wind field image will be output by the framework. Experiments show that the joint framework based on a multisensor possesses stronger ability to capture local feature and sequence feature than the traditional CNN or LSTM model.
\end{abstract}

\section{Introduction}

Airplane wake is a special atmospheric turbulence phenomenon which occurs in the whole flight process. Jet flow and wingtip wake are the two main part of airplane wake. To be more specific, wingtip wake consists of two whirlpools with opposite rotation directions, which lasts for tens of seconds to several minutes. In space, airplane wake is a long cylindrical distribution medium target behind the airplane. Generally, it reaches a hundred times of wingspan at the rear of the airplane, with a length of several kilometers, which belongs to a very strong turbulence. In 2004, the Federal Aviation Administration (FAA) defined wake vortex or wingtip wake as "Round air mass caused by the movement of wings in the air during the process of generating lift" (see Figure 1). When the airplane flies through the clouds, the water vapor particles will be stirred by a strong air flow, which looks like a vortex [1-5].

Actually, every airplane creates wake but the strength of the different airplane type is determined by many factors, including the weight, speed, wingspan (or rotor design) of the airplane, and the atmospheric conditions during the process. Part of the reason for wake generation is the same as airplane lift [6-8]. Based on the propulsion of the airplane engine and the configuration characteristics of the wing surface, the pressure difference will be created when the air flows through the wing surface. For the reason that the air pressure of the lower wing surface is higher than that of the upper wing surface, the lift force is formed. At the same time, the high-pressure air from the lower surface of the wing will tumble around the tip of the wing and then get into the low-pressure air above the wing, forming two vortices at the rear. However, the structure of the vortex is small, the two wake vortices will induce each other to eventually form a horseshoe-shaped vortex.

As the aviation industry has grown, the quantity of airplanes has increased sharply, which means that the flight interval between airplanes has been reduced. So the impact of wake on the flight safety and air traffic control has become crucial. Another side effect of airplane wake is restricting airport throughput. That means that decreased utilization of airspace and runways will make it impossible to increase 


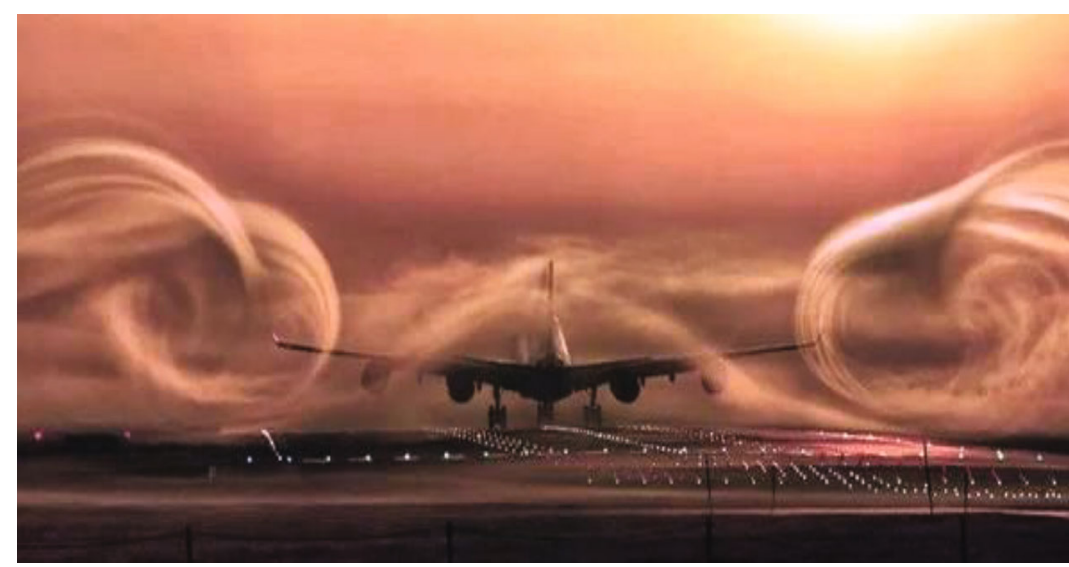

FIgURE 1: Airplane wake vortex.

airport capacity and cause flight delays. In general, the adverse effects of the wake will damage the aerodynamic performance of the rear machine. In serious cases, flight accidents will be triggered, especially in the takeoff and landing, due to the decrease of flight speed and the poor aerodynamic performance $[9,10]$.

With the development of navigation technology and information technology, experts propose that the utilization of airspace can be improved by reducing the wake spacing of airplanes. However, the primary task of reducing wake separation is to detect and identify the wake of the airplane. The key to solve these problems is to detect and identify the dissipative characteristics of airplane wake by means of technology. In order to achieve this, the development of sensor technology and deep learning technology will provide us with a feasible scheme. The convolutional neural network $(\mathrm{CNN})$ is considered to be one of the most widely used deep learning techniques such as target recognition and image processing, which can provide an idea of accurate eddy current identification based on a flow field image. With the addition of the CNN, the model can identify the flow field image and locate the wake accurately [11-16]. Concurrently, the long short-term memory (LSTM) network also has good performance in sequence feature extraction and data classification [17-19]. In addition, with the maturity of detection equipment technology, lidar has gradually become the most mature way to study airplane wake due to its high precision and high accuracy. At the same time, airport surveillance radar has been applied to airplane detection within the airport, which has also been proved to be effective in detecting and locating airplanes. The fusion of the two sensors can greatly improve the efficiency and accuracy of the wake location [20-31].

Therefore, this paper takes the detection and recognition of airplane wake in the near-Earth stage as the research content and detects the wind field in the flight channel of the airport with the help of Doppler lidar and a surveillance radar is used to obtain the real-time flight status of the airplane. Finally, deep learning technology is used to fuse information of different sensors and realize the accurate identification and prediction of the wake vortex. Effective identification of the wake vortex can improve flight safety and reduce the existing wake interval, so as to ensure flight safety. It is of great significance to the steady development of the civil aviation industry to reduce the second flight delay and relieve the capacity pressure of the airport.

\section{Preliminary}

As an important basis for studying the dissipation structure and characteristics of airplane wake, the field wind field measurement for wake can accumulate a large amount of measured data, which can be used to better mine the temporal and spatial characteristics of wake in different wind fields and through the auxiliary monitoring of the airport surveillance radar, so as to make the identification results more accurate, which is of great practical significance $[5,6,31-36]$.

Coherent Doppler lidar is the product of the combination of traditional radar technology and modern laser technology, which possesses the characteristics of high range and angle resolution, accurate position information, strong antijamming ability, and all-weather operation. Due to unique four-dimensional scattering distribution, we need to fully consider the precise change of the tangential velocity of the wake in space and time and the working characteristics of the lidar. The detection content of coherent Doppler lidar includes the radial velocity information, signal-tonoise ratio information, Doppler spectrum, data and other information. In view of the above requirements, the continuous development of radar technology provides technical possibility for the field wind field measurement.

In order to obtain the $3 \mathrm{D}$ scanning of a complex wind field and the accurate data of airplane wake, wind3d 6000 coherent Doppler lidar was selected to collect the wind field data in airports through enough tests (see Figure 2 and Table 1).

Wind3d 6000 radars were placed in several airports to detect the wind field changes in the side direction perpendicular to the airplane wake vortices using the RHI scanning mode. By setting the change period of the pitch angle, we can get the wind field data image $H^{k}=\left(a_{i j}\right)_{m \times n}$ in polar coordinates as shown in Figure 3, where $a_{i j}$ is the radial 


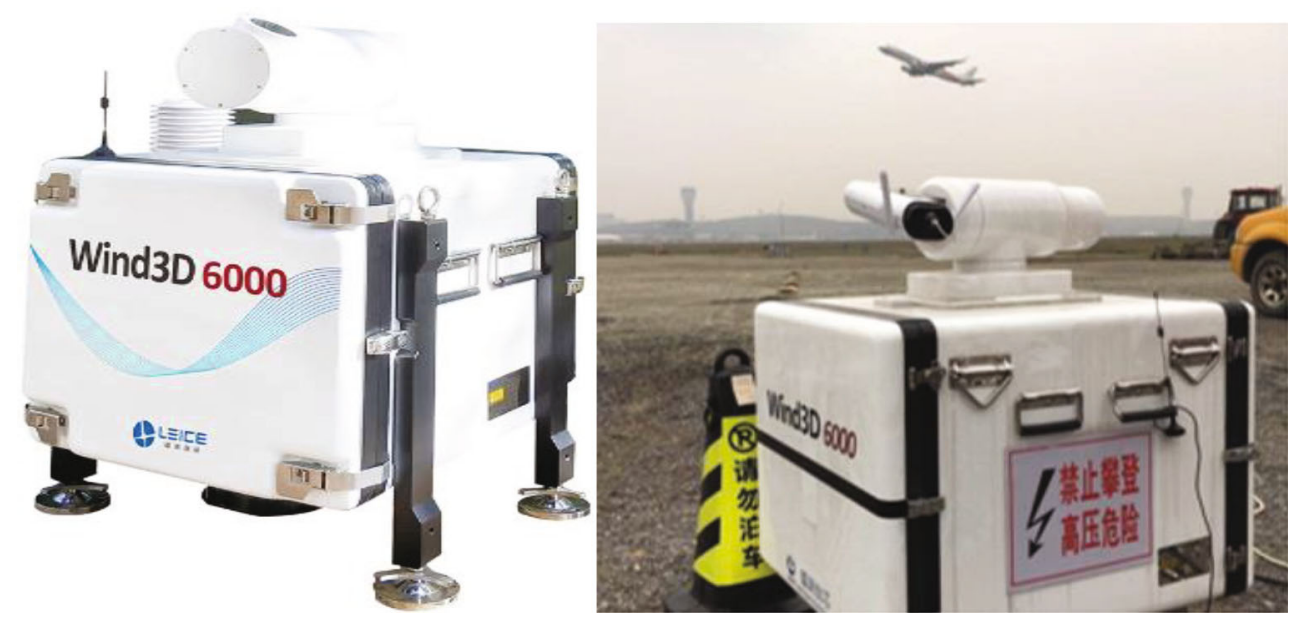

FIGURE 2: Wind3D 6000.

TABLE 1: Radar parameters.

\begin{tabular}{lc}
\hline Index item & Parameter \\
\hline Laser wavelength & $1.5 \mu \mathrm{m}$, invisible, and safe for the \\
human eyes & $45 \mathrm{~m} \sim 6000 \mathrm{~m}$ \\
Radial detection range & $15 \mathrm{~m} / 30 \mathrm{~m} / \mathrm{user}$ setting \\
Radial distance resolution & $1 \mathrm{~Hz} \sim 10 \mathrm{~Hz},-37.5 \mathrm{~m} / \mathrm{s} \sim 37.5 \mathrm{~m} / \mathrm{s}$ \\
$\begin{array}{l}\text { Data refresh rate } \\
\text { Wind speed }\end{array}$ & $\leq 0.1 \mathrm{~m} / \mathrm{s}$ \\
measurement accuracy & Fixed point/DBS/VAD/PPI/RHI/ \\
Ccanning servo accuracy & Cappi script programming \\
\hline
\end{tabular}

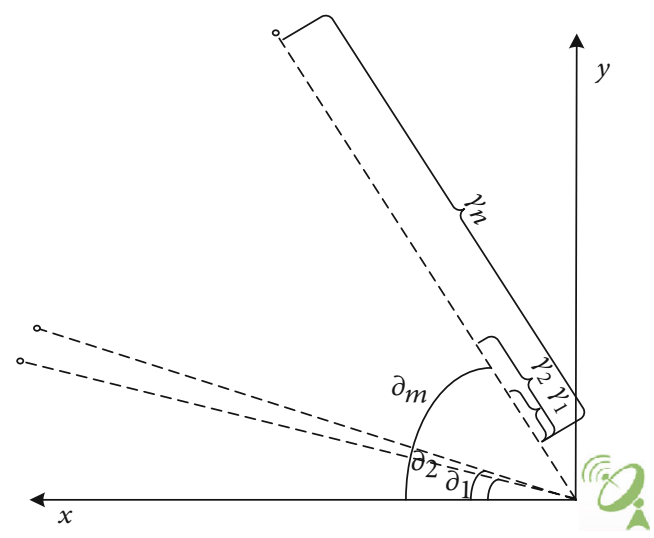

FIgURE 3: Detection principle in polar coordinates.

velocity of the wind field in different locations $\left(i=\partial_{1}\right.$, $\partial_{2}, \cdots, \partial_{m}$ represents pitch angles and $j=\gamma_{1}, \gamma_{2}, \cdots, \gamma_{n}$ represents distances to range radar) [37-42].

Meanwhile, the airport surveillance radar can reliably monitor and track within $75-110 \mathrm{~km}$ of the airport and the flight altitude is less than $6000 \mathrm{~m}$ which can help coherent Doppler lidar to better obtain the flight state and airplane position (see Figure 4).
It is the fact that the data of the RHI wind field detected by the radar is easily interfered by the external atmospheric environment and the clutter of the equipment itself, in order to eliminate the interference of the signal echo in the transmission and detection process and to strengthen the tail vortex Doppler characteristics of the data. It is necessary to preprocess the data and eliminate the useless noise data and interference data [43-48]. At the same time, the surveillance radar data also needs corresponding spatiotemporal alignment and filtering.

\section{Methodology}

According to the characteristics of data generated by a multisensor, it is obvious that the data is fusion information composed of regional radial wind speed, wind field parameters, flight status, and airplane positioning. Supposing that it is necessary to identify the wake vortices in the wind field, the model needs to possess strong recognition ability for local features and sequence features.

In this paper, we employ a parallel DL frameworkLSTM and CNN-to capture fusion information in wake vortex recognition. In this section, we first present a brief review of the traditional LSTM and CNN.

3.1. LSTM. Long short-term memory (LSTM) networks, a special RNN network, are designed to solve the problem of long dependence. The network was introduced by Hochreiter \& Schmidhuber (1997) and has been improved and popularized by many people. Their work has been used to solve a variety of problems; until now, it has been widely used.

The core of LSTM is a cell state, which is represented by the horizontal line through the cell. Cells are in the same state as conveyor belts. It runs through the whole cell but has only a few branches, which can ensure that the information flows through the RNNs unchanged.

As a kind of deep learning model, long-term and shortterm memory networks have a chain structure. Time stamps are used to connect cells, which can effectively enhance the memory ability of memory cells. Each memory cell is 


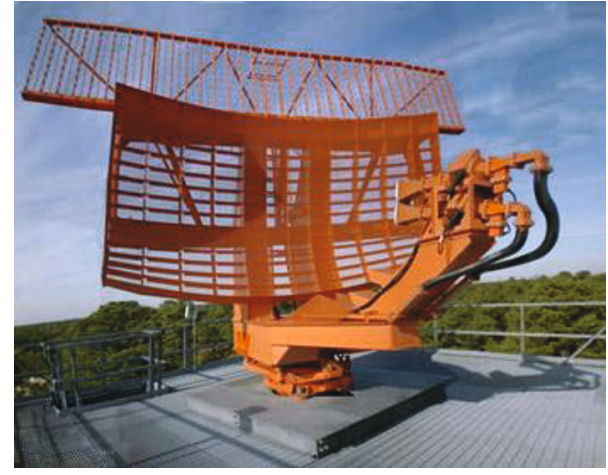

FiguRE 4: Surveillance radar.

controlled by three parts: input gate, forgetting gate, and output gate. The input gate is mainly responsible for saving the input vector to the memory cell. In this process, some information will be deleted selectively by the forgotten door. After some processing, the new information will become the input information of the next memory cell and output the final result through the output gate after several iterations. The whole process schematic diagram of the LSTM model is shown in the figure below (see Figure 5).

3.2. CNN. As a convolutional neural network of the feedforward neural network, the connection mode of its neurons is inspired by the visual cortex of animals, with the least number of multilayer perceptron recognition variants. LeNet is an early convolutional neural network, which is Yann Lecun's successful work after many iterations. The convolution neural network can not only complete the work of the reading postal code and numbers but also accurately process images and recognize video data. Among them, the structure of the convolution neural network is mainly composed of four parts: the convolution layer, activation function, pooling layer, and fully connected layer.

3.2.1. Convolution Layer. The convolution layer is a kind of mathematical operation on the input variables. For example, convolution of variables will produce a third new variable. In the process of feature extraction, multiple filters are needed because the weights of different filters and data windows are different and fixed, so the content of each filter is different.

3.2.2. Activation Function. The activation function is to reflect the more complex mapping relationship in the model, that is, the acquired local features are mapped to the new feature map. At present, the common activation functions are sigmoid, $\tan h$, and ReLu. But the convolution layer usually uses ReLu as its activation function, because it can reduce the overfitting problem and make the calculation easier (see Figure 6).

3.2.3. Pool Layer. The pooling layer is a process of sample discretization, that is, to reintegrate the input sample data and reduce the dimension without reducing the sample characteristics. The most common pooling processes use

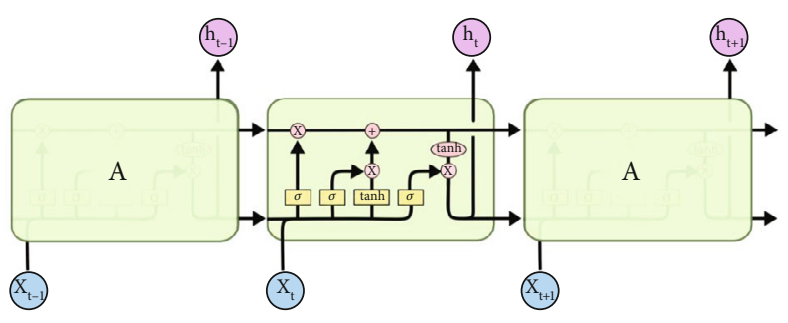

FIGURE 5: Structure of LSTM.

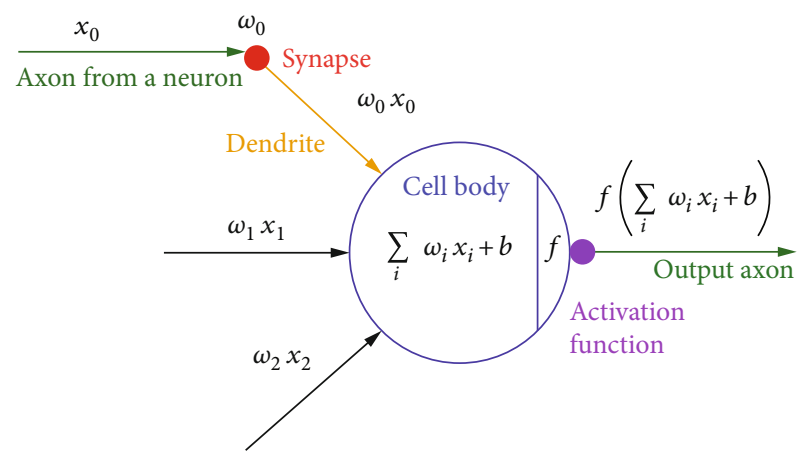

Figure 6: Activation function.

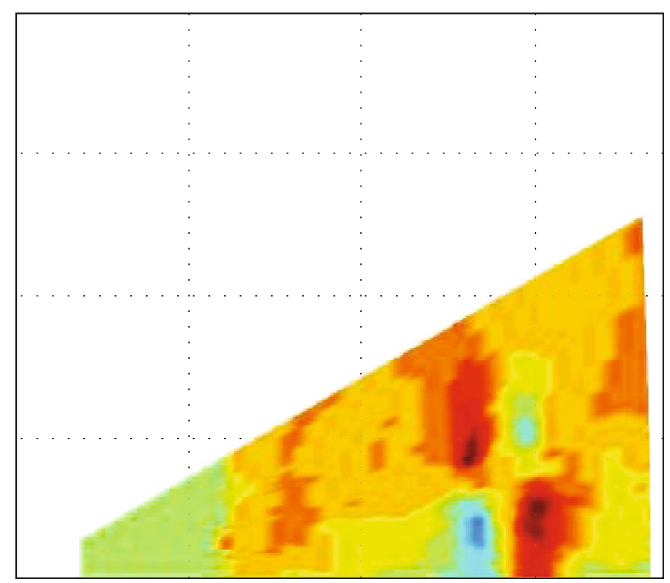

FIGURE 7: Wake vortex image in coherent Doppler lidar.

an abstract form to reduce the number of parameters and the difficulty of calculation.

3.2.4. Random Deactivation. As an artificial intelligence deep learning model, it has the advantages that the traditional model cannot compare; but because of too many parameter settings, it is easy to lead to model overfitting. When the problem of overfitting is solved by random deactivation (dropout layer), it is not necessary to add new constraints or train more models, only to change the eigenvalue of the hidden layer to 0 according to a certain proportion.

3.2.5. Full Connection Layer. As a multilayer perceptron, the all-connected layer refers to the connection between each 


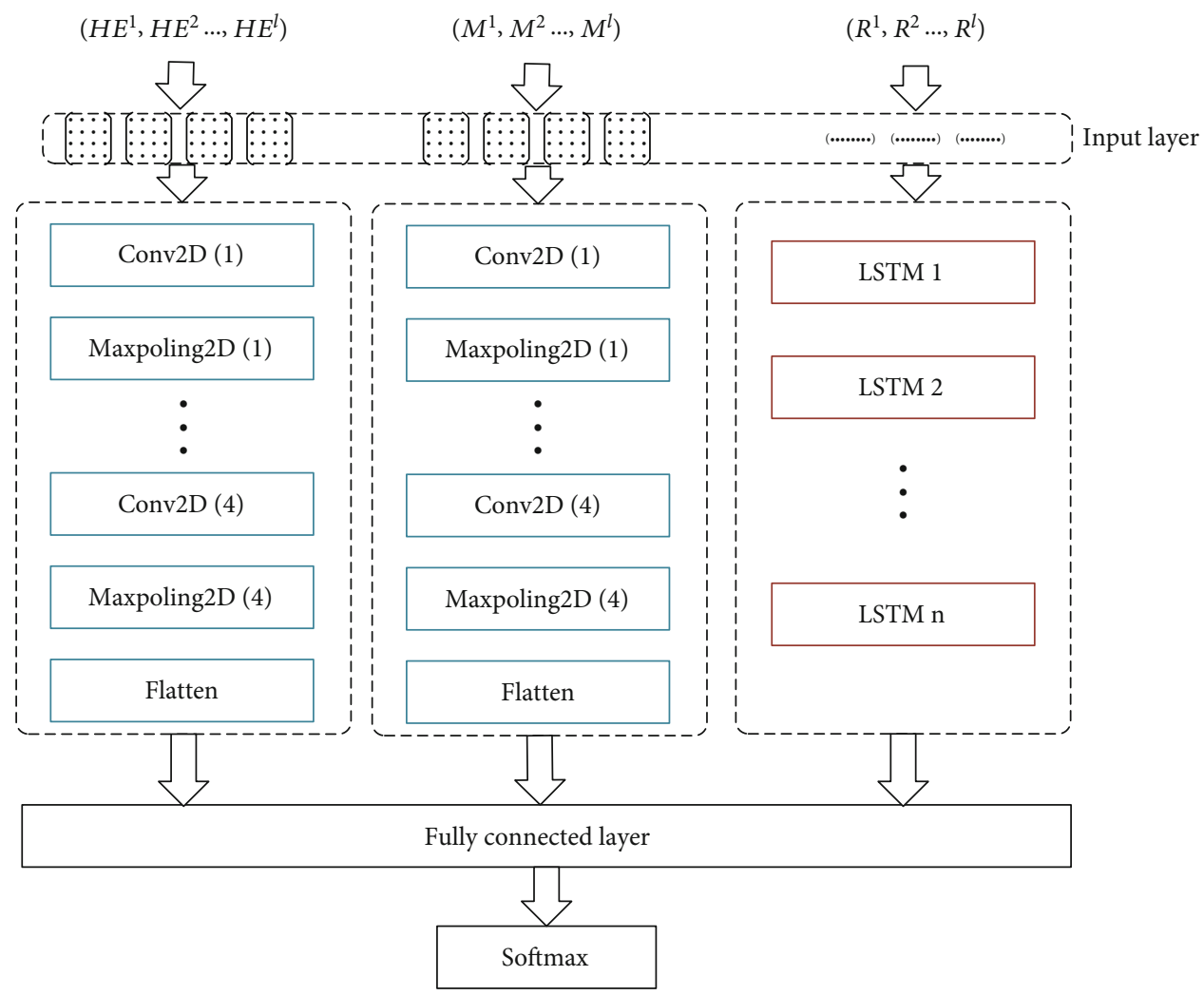

FiguRE 8: Framework of CNN-LSTM.

neuron in the former layer and each neuron in the latter layer. At the same time, the full connected layer calculates the hidden layer of the model through the linear function and obtains the final output results.

3.3. Combination of CNN and LSTM. Radar data usually appear in polar coordinates (see Figure 7). Therefore, the radar image must be accompanied by periodic characteristics and local characteristics. Focusing on the joint recognition of temporal and image features, the CNN and LSTM, respectively, show good performance in their respective fields. Local features will be better captured by CNN, and temporal features will be more suitable for LSTM. Hence, we will use a combined structure to recognize the wake vortex by a multisensor.

The spatial features in the radar image will be captured by the CNN in this paper. Firstly, due to the dimension requirement of the $\mathrm{CNN}$ for input variables, the wind field matrix produced by one radar scanning cycle will be expanded to the $n \times n$ matrix form which fills in the missing position with $0\left(H^{k}=\left(a_{i j}\right)_{m \times n} \longrightarrow \mathrm{HE}^{k}=\left(a_{i j}\right)_{n \times n}\right)$. Supposing that we need to recognize the wake vortex in $k$ th wind field images, the wake vortex will exhibit some special local characteristics which will trigger a series of wind field evolution. For these regular local features, the CNN will show a good learning ability.

In this paper, LSTM is applied to the learning of sequence features. For the wind field image, when the airplane wake is detected in the region, the wind field intensity around the wake vortex will show an obvious upward trend.
In order to make the model better grasp this trend, this paper deals with the radar data according to the sequence characteristic. We can generate the variance sequence of the radial wind velocity of each radar record point at different pitch angles, and these sequences will contain wake vortex characteristics so that the LSTM model can grasp them.

$$
\begin{aligned}
& R_{j}^{k}=\sum_{i}\left(a_{i j}-\mathrm{EX}_{j}\right)^{2}, \\
& R^{k}=\left(R_{1}^{k}, R_{1}^{k}, \cdots, R_{n}^{k}\right),
\end{aligned}
$$

where $R^{k}$ is the variance sequence of the radial wind velocity and $j=\gamma_{1}, \gamma_{2}, \cdots, \gamma_{n}$ are data record points at different distances. $\mathrm{EX}_{j}$ represents the mean value of radial wind velocity of record point $j$ at different pitch angles $i=\partial_{1}, \partial_{2}, \cdots, \partial_{m}$.

Furthermore, in order to better integrate airport surveillance radar information, we transform the spatial coordinate system of the airport surveillance radar into a $200 \times 200$ grid network, in which the grid accuracy is $20 \mathrm{~m}$, where $M^{k}=$ $\left(b_{i j}\right)_{200 \times 200}$ represents the airplane positioning matrix.

The applied combination of the CNN and LSTM is used to capture the spatial features $\mathrm{HE}^{k}, M^{k}$ and the sequence features $R^{k}=\left(R_{1}^{k}, R_{1}^{k}, \cdots R_{n}^{k}\right)$, where $\mathrm{HE}^{k}$ reflects the local change of regional wind field intensity, $M^{k}$ can infer the accurate spatial position of airplane and wake, and $R^{k}$ represents the difference characteristics of the wind field sequence. The 

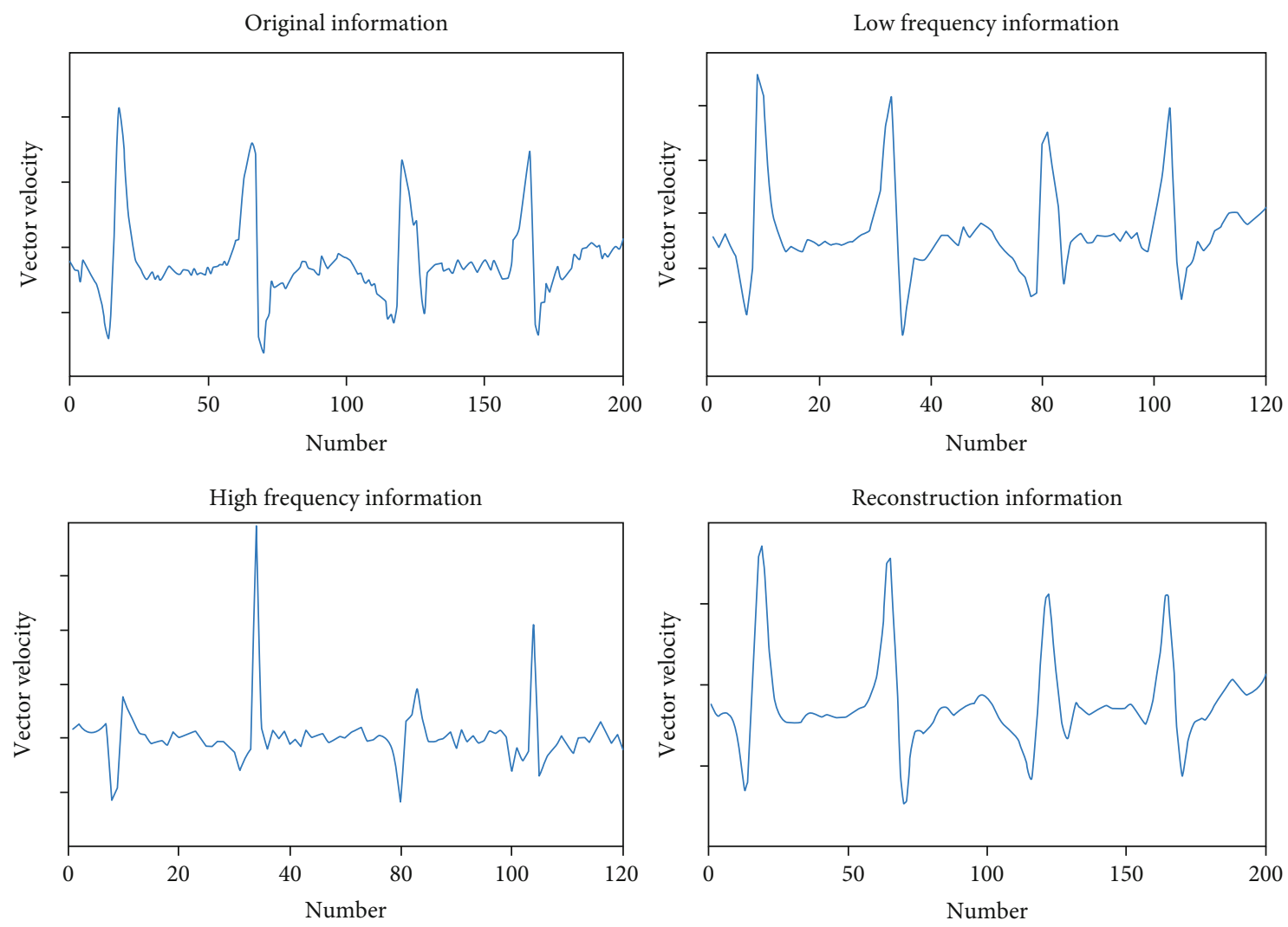

FIGURE 9: Data denoising results.

fusion of three spatial and sequential variables can greatly improve the detection accuracy of the airplane wake vortex.

Firstly, in order to realize the recognition of variable characteristics, the model will be divided into three channels for learning. Secondly, we add a full connection layer to achieve the fusion of local features and temporal features. Finally, a softmax layer is set to achieve information recognition and classification (see Figure 8).

\section{Experiments and Results}

4.1. Datasets. In this paper, the real-time wind field data has been generated by coherent Doppler lidars which have been located at Qingdao Liuting Airport (TAO) and Chengdu Shuangliu Airport (CTU) from Aug 16, 2018, to Oct 10, 2018. In addition, the airport surveillance radar data is also matched with the wind field data. In particular, various types of civil airplanes will take off and land at these airports, which will bring more promotion for the recognition accuracy and adaptability of the model.

Due to the low SNR of the echo signal, in order to reduce the interference of the high-frequency clutter in the sensor signal and retain the characteristics of the wake vortex contained in the low-frequency signal, this paper uses the discrete wavelet transform to process the signal. The discrete wavelet basis function is defined as follows:

$$
\Psi_{b, c}(t)=\frac{1}{\sqrt{b}} \Psi\left(\frac{t-c}{b}\right) .
$$

TABLE 2: CNN parameters.

\begin{tabular}{lc}
\hline Layer (type) & Parameter \\
\hline $\begin{array}{l}\text { conv2d_1 (Conv2D) } \\
\text { max_pooling2d_1 }\end{array}$ & $(32,(3,3)$, activation = "ReLu") \\
onv2d_2 (Conv2D) & $(2,2)$ \\
max_pooling2d_2 & $(64,(3,3)$, activation = "ReLu") \\
conv2d_3 (Conv2D) & $(64,(3,3)$, activation = "ReLu") \\
max_pooling2d_3 & $(2,2)$ \\
conv2d_4 (Conv2D) & $(64,(3,3)$, activation $=$ "ReLu") \\
dense_1 (dense) & 64 \\
dense_2 (dense) & 1 \\
\hline
\end{tabular}

TABLE 3: LSTM parameters.

\begin{tabular}{lc}
\hline Layer (type) & Parameter \\
\hline dense_1 (dense) & 64 \\
dense_2 (dense) & 32 \\
Softmax & 2 \\
\hline
\end{tabular}



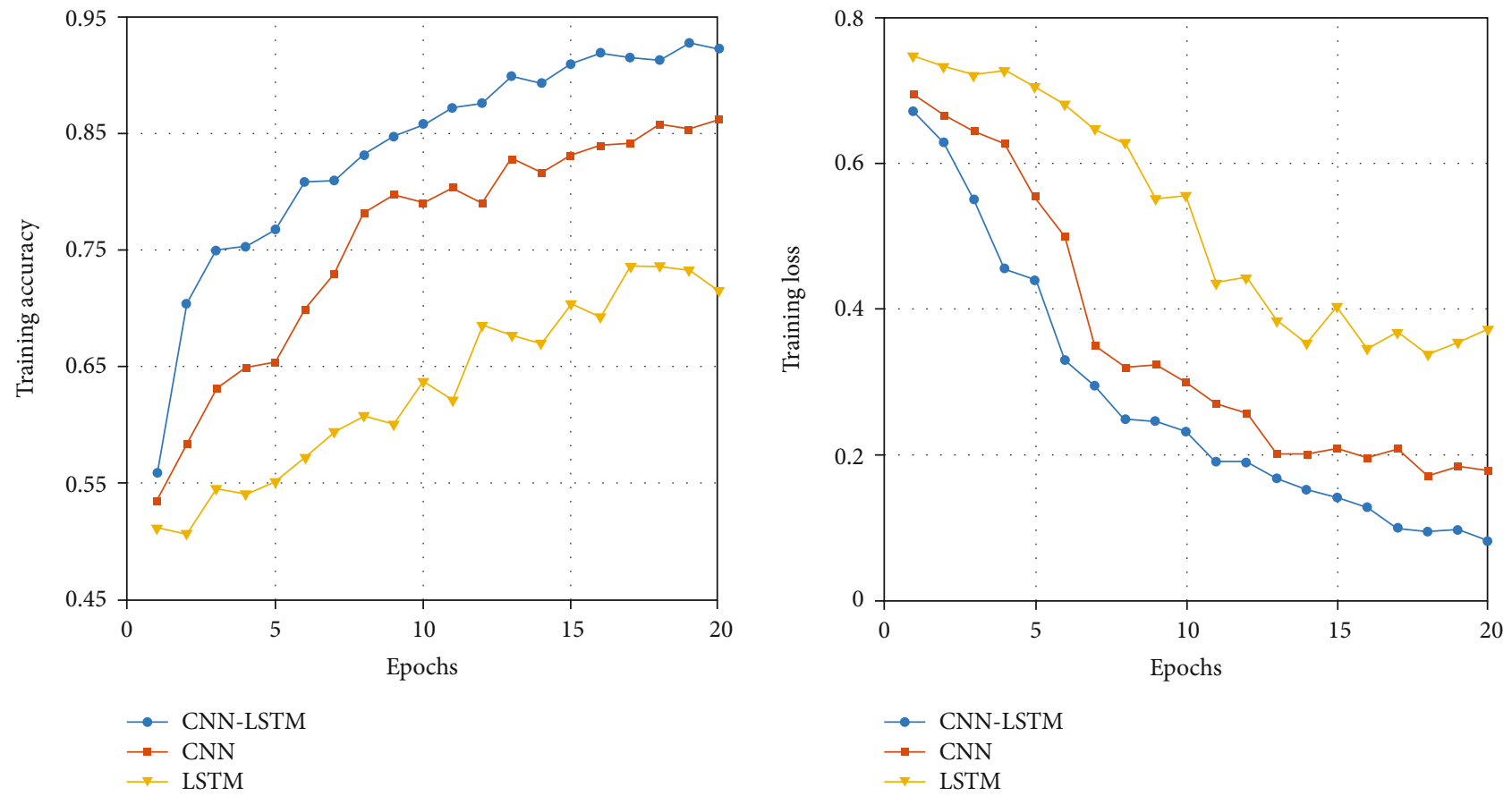

Figure 10: Training accuracy and loss.
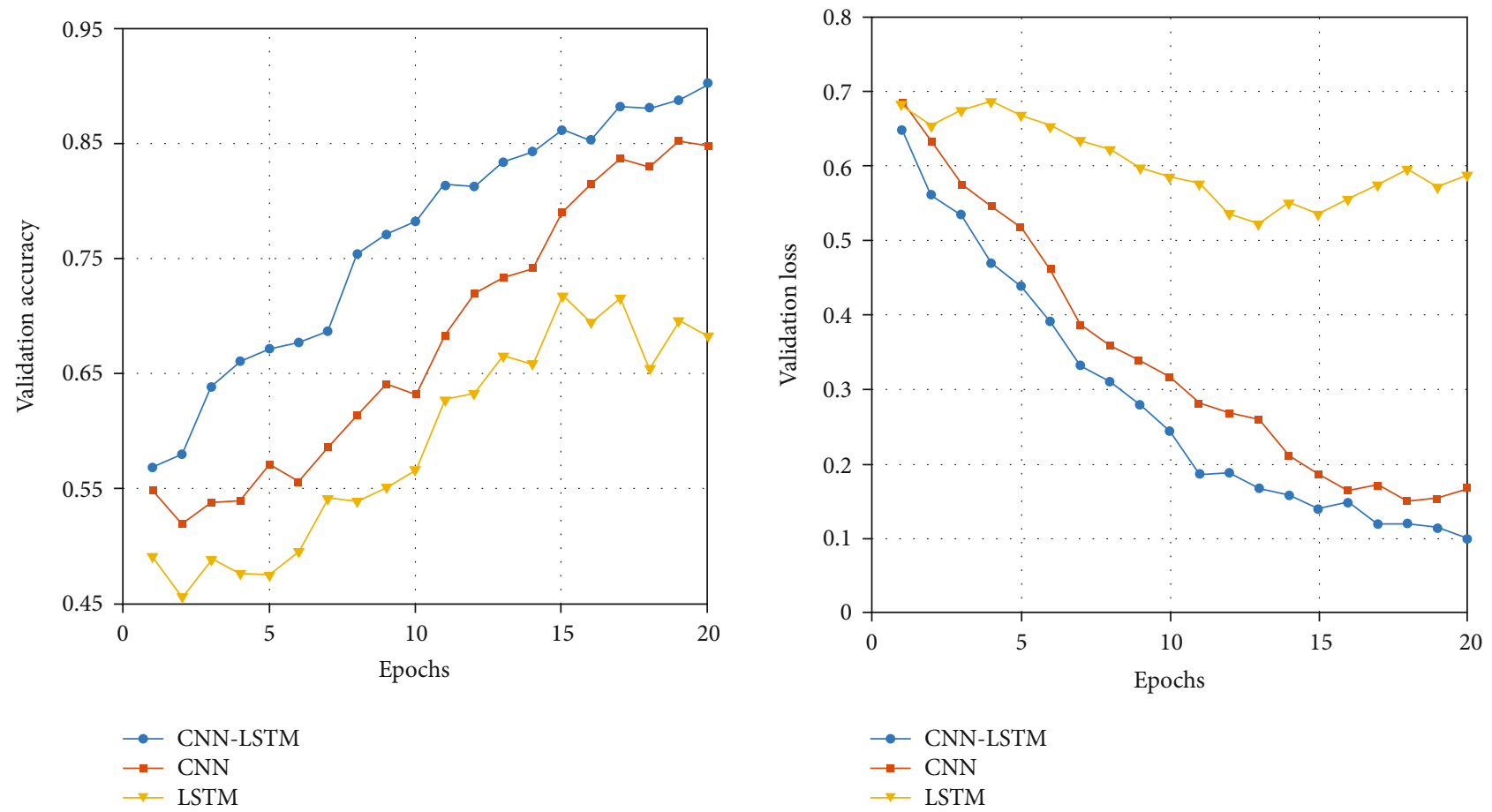

Figure 11: Validation accuracy and loss.

$\Psi_{a, b}(t)$ is wavelet basis, $b$ is the scale factor, and $c$ is the time translation factor.

In this paper, the high-frequency information and lowfrequency information are separated with the wavelet denoising method:

It can be seen that after denoising, the signal-to-noise ratio of data has been significantly improved in Figure 9, which indicates that the data quality after denoising has been significantly improved.

For airport surveillance radar data, it includes the airplane flight number, registration number, wake level, longitude and latitude coordinates, and other parameters. However, track correlation and track filtering need to be carried out for all airplanes within the range based on the 
TABLE 4: Predictive performance comparison.

\begin{tabular}{lcccc}
\hline Model & Training accuracy & Validation accuracy & Training loss & Validation loss \\
\hline CNN-LSTM & 0.922 & 0.901 & 0.083 & 0.099 \\
CNN & 0.862 & 0.847 & 0.179 & 0.167 \\
LSTM & 0.716 & 0.682 & 0.373 & 0.587 \\
\hline
\end{tabular}

airplane registration numbers, so as to integrate them into relatively complete and smooth independent tracks.

In addition, the track coordinates and wind field coordinates of each aircraft are spatiotemporal aligned and standardized to ensure the real-time matching of the track and wind field.

4.2. Results. In this paper, the dataset will be partitioned into two parts: the first part is training data (40 days) and the second part is test data (16 days). The employed CNNLSTM with full variables are trained on the training set and validated on the test set. Meanwhile, two CNN networks and a LSTM network with the same topology are adopted to train and test, respectively, where the wind field matrix $\mathrm{HE}^{k}$, the airplane positioning matrix $M^{k}$, and the sequence features $R^{k}=\left(R_{1}^{k}, R_{1}^{k}, \cdots R_{n}^{k}\right)$ are used as the input variable of CNN and LSTM. The definitions of two models are shown in Tables 2 and 3 as follows:

Figures 10 and 11 show the learning performance of the CNN-LSTM, CNN, and LSTM on the training set, where the training accuracy and validation loss after each training epoch are recorded for all models. It can be observed that the training accuracy of CNN-LSTM increases faster than other models in the initial 5 epochs, and similarly, the training loss of CNN-LSTM also showed a faster decline rate. The training results indicate that CNN-LSTM has a higher convergence speed and learning performance than traditional model frameworks in wake recognition training. In addition, the training process of the LSTM network is relatively difficult due to the single information in variables.

In the following model validation process, the $\mathrm{CNN}$ LSTM shows more excellent recognition ability than other models for the wake vortex in the wind field, which benefits from the fusion of sequence features and local features (see Figures 10 and 11). Moreover, the CNN also shows good image recognition ability, and on the contrary, the LSTM has some disadvantages for image features (see Table 4).

\section{Conclusions}

This paper presents a joint framework to detect and identify airplane wake vortices in the wind field based on a multisensor. The coherent Doppler radar has been proved to have excellent performance in the field of wind field detection. At the same time, airport surveillance radar data is innovatively introduced and fused with wind field data, where the integration of a track enables the model to capture the spatiotemporal variation characteristics of wake vortex easier. Besides that, the employed CNN-LSTM network can capture local features and sequence features simultaneously, which is especially desirable for image recognition with time series features. Therefore, aiming at the problem of wake recognition in the wind field, a fusion framework composed of CNN-LSTM and a multisensor has been regarded as an ideal solution. To validate the effectiveness of the proposed framework, the wind3d 6000 coherent Doppler radars have been installed to continuously monitor the wind field changes over the airport runway in several airports. After the discrete wavelet denoising, the fusion information from the multisensor is transformed into three input forms of the CNNLSTM model-the wind field matrix and the variance sequence. Through the training and validation of the CNN-LSTM model, the recognition accuracy of this framework for the wake vortex in wind field reaches $90 \%$, which is proved to have high practical value and research significance.

Furthermore, several future work can be considered in this study. Firstly, the model variables can be further integrated by introducing the time dimension. By combining the continuous wind field images into a continuous segment, the spatiotemporal information contained in variables will be significantly improved. It is worth noting that the deep learning framework also needs to be expanded on this basis, which will transform time variables and space variables into spatiotemporal variables. In addition, the influence factors of different meteorological conditions and wind conditions on the wake vortex can be introduced, which may greatly improve the recognition accuracy of the model.

This method provides a new idea for wake detection under multisensor and multisource data and innovatively realizes the real-time fusion and accurate prediction of the track and wake vortex.

\section{Data Availability}

The data used to support the findings of this study are available from the corresponding author upon request.

\section{Conflicts of Interest}

We declare that there is no conflict of interest.

\section{Acknowledgments}

This work was supported by joint funds of the National Science Foundation of China and the Civil Aviation Administration (grant number U1733203), Sichuan Science and Technology Project (grant number 2021JDRC0083), and funds of Civil Aviation Flight University of China (J2021069 and J2020-075). 


\section{References}

[1] S. Lang, J. Tittsworth, W. H. Bryant et al., Progress on an ICAO Wake Turbulence Re-Categorization EffortAIAA Atmospheric and Space Environments Conference, p. 7682, Toronto, Ontario, Canada, 2010.

[2] J. Babcock and R. Lind, "Aeroelastic Effects of Wing Stiffness on the Flight Dynamics of a MAV," in AIAA Atmospheric Flight Mechanics Conference, p. 4400, Minneapolis, Minnesota, 2012.

[3] T. Leweke and C. H. K. Williamson, "Experiments on longwavelength instability and reconnection of a vortex pair," Physics of Fluids, vol. 23, no. 2, p. 024101, 2011.

[4] I. Hennemann and F. Holzäpfel, "Large-Eddy simulation of aircraft wake vortex deformation and topology," Journal of Aerospace Engineering, vol. 225, no. 12, pp. 1336-1350, 2011.

[5] R. E. Loucel and J. D. Crouch, "Flight-simulator study of airplane encounters with perturbed trailing vortices," Journal of Airplane, vol. 42, no. 4, pp. 924-931, 2005.

[6] D. Vechtel, "Flight simulator study on the influence of vortex curvature on wake encounter hazard using LES wind fields," Aeronautical Journal, vol. 116, no. 1177, pp. 287-302, 2012.

[7] D. Bieniek and R. Luckner, "Simulation of airplane encounters with perturbed vortices considering unsteady aerodynamic effects," Journal of Airplane, vol. 51, no. 3, pp. 705-718, 2014.

[8] G. Höhne, M. Fuhrmann, and R. Luckner, "Kritische Einflugbedingungen in eine Wirbelschleppe," Aerospace Science and Technology, vol. 8, no. 8, pp. 689-701, 2004.

[9] T. Sarpkaya, "New model for vortex decay in the atmosphere," Journal of Airplane, vol. 37, no. 1, pp. 53-61, 2000.

[10] T. Gerz, F. Holzäpfel, and D. Darracq, "Commercial aircraft wake vortices," Progress in Aerospace Science, vol. 38, no. 3, pp. 181-208, 2002.

[11] Y. LeCun, L. Bottou, Y. Bengio, and P. Haffner, "Gradientbased learning applied to document recognition," Proceedings of the IEEE, vol. 86, no. 11, pp. 2278-2324, 1998.

[12] A. Krizhevsky, I. Sutskever, and G. E. Hinton, "Imagenet classification with deep convolutional neural networks," Advances in Neural Information Processing Systems, vol. 25, no. 2, pp. 1097-1105, 2012.

[13] K. Simonyan and A. Zisserman, "Very deep convolutional networks for large-scale image recognition," 2014, http://arxiv.org/abs/1409.1556.

[14] C. Szegedy, W. Liu, Y. Q. Jia et al., "Going deeper with convolutions," in Proceedings of the IEEE Conference on Computer Vision and Pattern Recognition, pp. 1-9, New Orleans, LA, USA, 2015.

[15] K. He, X. Zhang, S. Ren, and J. Sun, "Deep residual learning for image recognition," in Proceedings of the IEEE Conference on Computer Vision and Pattern Recognition, pp. 770-778, New Orleans, LA, USA, 2016.

[16] J. Long, E. Shelhamer, and T. Darrell, "Fully convolutional networks for semantic segmentation," in Proceedings of the IEEE Conference on Computer Vision and Pattern Recognition (CVPR), pp. 3431-3440, New Orleans, LA, USA, 2015.

[17] G. L. Tian, S. Zhou, G. X. Sun, and C. C. Chen, "A novel intelligent recommendation algorithm based on mass diffusion," Discrete Dynamics in Nature and Society, vol. 2020, Article ID 4568171, 9 pages, 2020.

[18] S. Bin and G. Sun, "Matrix factorization recommendation algorithm based on multiple social relationships," Mathemati- cal Problems in Engineering, vol. 2021, Article ID 6610645, 8 pages, 2021.

[19] S. Hochreiter and J. Schmidhuber, "Long short-term memory," Neural Computation, vol. 9, no. 8, pp. 1735-1780, 1997.

[20] X. Ma, Z. Tao, Y. Wang, H. Yu, and Y. Wang, "Long shortterm memory neural network for traffic speed prediction using remote microwave sensor data," Transportation Research Part C: Emerging Technologies, vol. 54, pp. 187-197, 2015.

[21] R. Zhao, R. Yan, J. Wang, and K. Mao, "Learning to monitor machine health with convolutional bi-directional LSTM networks," Sensors, vol. 17, no. 2, p. 273, 2017.

[22] S. Rahm, I. Smalikho, and F. Kopp, "Characterization of airplane wake vortices by airborne coherent Doppler lidar," Journal of Airplane, vol. 44, no. 3, pp. 799-805, 2007.

[23] S. Rahm and I. Smalikho, "Airplane wake vortex measurement with airborne coherent Doppler lidar," Journal of Airplane, vol. 45, no. 4, pp. 1148-1155, 2008.

[24] M. Keane, D. Buckton, M. Redfern et al., "Axial detection of aircraft wake vortices using Doppler lidar," Journal of Airplane, vol. 39, no. 5, pp. 850-861, 2002.

[25] F. Holzäpfel, "Probabilistic two-phase wake-vortex decay and transport model," J. Airplane, vol. 40, no. 2, pp. 323-331, 2003.

[26] D. C. Burnham and J. N. Hallock, "Decay characteristics of wake vortices from jet transport airplane," J. Airplane, vol. 50, no. 1, pp. 82-87, 2013.

[27] M. J. Pruis, D. P. Delisi, D. Jacob, and D. Y. Lai, "Summary of NASA wake and weather data collection. Memphis International Airport. 2013-2015," in 8th AIAA Atmospheric and Space Environments Conference, p. 3274, Washington, DC, 2016.

[28] F. Holzäpfel, A. Stephan, T. Heel, and S. Körner, "Enhanced wake vortex decay in ground proximity triggered by plate lines," Aircraft Engineering and Aerospace Technology, vol. 88, no. 2, pp. 206-214, 2016.

[29] G. F. Switzer and F. H. Proctor, Numerical Study of Wake Vortex Behavior in Turbulent Domains with Ambient Stratification, AIAA, 2000.

[30] D. C. Lewellen and W. S. Lewellen, "The effects of Aircraft wake dynamics on contrail development," Atmospheric Sciences, vol. 58, no. 4, pp. 390-406, 2001.

[31] I. Hennemann and F. Holzäpfel, "Large-eddy simulation of airplane wake vortex deformation and topology," Proceedings of the Institution of Mechanical Engineers, Part G, vol. 225, no. 12, pp. 1336-1350, 2011.

[32] A. Stephan, F. Holzäpfel, and S. Zholtovski, "The effect of gusts on aircraft wake vortices," Aircraft Engineering and Aerospace Technology, vol. 89, no. 5, pp. 692-702, 2017.

[33] S. M. Mackey, F. Y. Wang, H. Wassaf, and M. Soares, "Comparison between arrival and departure wake vortex statistics near the ground," in Proc. 1st CEAS European Air and Space Conf.,-Century Perspectives, pp. 1049-1054, Berlin, Deutschland, 2007.

[34] D. Vechtel, "Simulation study of wake encounters with straight and deformed vortices," Aeronautical Journal, vol. 120, no. 1226, pp. 651-674, 2016.

[35] D. Bieniek, R. Luckner, I. De Visscher, and G. Winckelmans, "Simulation methods for aircraft encounters with deformed wake vortices,” Airplane, vol. 53, no. 6, pp. 1581-1596, 2016.

[36] D. Bieniek and R. Luckner, "Simulation of Aircraft encounters with perturbed vortices considering unsteady aerodynamic effects," Airplane, vol. 51, no. 3, pp. 705-718, 2014. 
[37] C. Schwarz and K.-U. Hahn, "Full-Flight Simulatorstudie zur Untersuchung von Wirbelschleppen- Gefahrdungsraumen," Aerospace Science and Technology, vol. 10, no. 2, pp. 136143, 2006.

[38] R. Luckner, "Modeling and simulation of wake vortex encounters: state-of-the-art and challenges," in AIAA Modeling and Simulation Technologies Conference, p. 4633, Minneapolis, Minnesota, 2012.

[39] F. Holzäpfel, "Analysis of potential wake vortex encounters at a major European airport," Aircraft Engineering and Aerospace Technology, vol. 89, no. 5, pp. 634-643, 2017.

[40] R. Luckner, G. Höhne, and M. Fuhrmann, "Gefahrdungskriterien fur das Durchfliegen von Wirbelschleppen wahrend des Landeanfluges," Aerospace Science and Technology, vol. 8, no. 8, pp. 673-687, 2004.

[41] N. P. Schmitt, W. Rehm, T. Pistner, P. Zeller, H. Diehl, and P. Nave, "Der bordgetragene AWIATOR LIDAR TurbulenzSensor," Aerospace Science and Technology, vol. 11, no. 7-8, pp. 546-552, 2007.

[42] J. Ehlers, D. Fischenberg, and D. Niedermeier, "Wake impact alleviation control based on wake identification," Airplane, vol. 52, no. 6, pp. 2077-2089, 2015.

[43] J. Cheng, J. Tittsworth, W. Gallo, and A. Awwad, "The development of wake turbulence recategorization in the United States," in 8th AIAA Atmospheric and Space Environments Conference, p. 3434, Washington, DC, 2016.

[44] J. N. Hallock, G. C. Greene, J. A. Tittsworth, P. D. Strande, and F. Y. Wang, "Use of simple models to determine wake vortex categories for new airplane," in 7th AIAA Atmospheric and Space Environments Conference, p. 3172, Dallas, TX, 2015.

[45] D. A. Hinton, Description of Selected Algorithms and Implementation Details of a Concept-Demonstration Airplane Vortex Spacing System (AVOSS), NASA TM-211027, 2001.

[46] F. Holzäpfel, K. Dengler, T. Gerz, and C. Schwarz, "Prediction of dynamic pairwise wake vortex separations for approach and landing," in 3rd AIAA Atmospheric Space Environments Conference, pp. 15-16, Honolulu, Hawaii, 2011.

[47] I. Sölch, F. Holzäpfel, F. Abdelmoula, and D. Vechtel, "Performance of on-board wake vortex prediction systems employing various meteorological data sources," Airplane, vol. 53, no. 5, pp. 1505-1516, 2016.

[48] H. Frank, "Airplane wake-vortex evolution in ground proximity: analysis and parameterization," AIA Journal, vol. 45, no. 45 , pp. $218-227,2007$. 